\title{
Recurrent cardiac intimal sarcoma misdiagnosed as a myxoma or malignant transformation of a cardiac myxoma?
}

\author{
rodolphe Durieux ${ }^{1}$, Vincent Tchana-Sato ${ }^{2}$, Jean-Paul Lavigne ${ }^{1}$, marc Radermecker ${ }^{2}$, Marie \\ Moonen $^{2}$, Irène Scagnol ${ }^{2}$, Christine Gennigens ${ }^{2}$, and Jean Olivier Defraigne ${ }^{2}$ \\ ${ }^{1}$ University Hospital of Liege Cardiovascular Surgery \\ ${ }^{2}$ University Hospital of Liège
}

August 7, 2020

\begin{abstract}
Cardiac intimal sarcoma are extremely rare and aggressive primary malignant cardiac tumors. Here, we reported the case of a young man initially operated for a tumor of the left atrium, causing a dynamic obstruction of the mitral valve and (mis)diagnosed as a myxoma at the histopathological analysis. Patient presented a local recurrence at 3 months and was reoperated. Pathology revealed this time the presence of an intimal sarcoma. Patient received adjuvant chemotherapy. Despite a good local control, the one-year follow-up PET scan revealed the presence of a metastasis in the left adrenal gland that was surgically resected. This paper aims to highlight the risk of misdiagnosis in case of cardiac tumors, the hypothetical concept of malignant transformation of a cardiac myxoma, the aggressive course of the extremely rare cardiac intimal sarcoma and the therapeutic modalities available to treat this pathology.
\end{abstract}

\section{Introduction}

Primary cardiac tumors are rare and the majority of these (75\%) are benign (1). Myxomas represent the most frequent benign cardiac tumors. Among the malignant primary cardiac tumor, sarcomas are the most frequently encountered. Intimal sarcoma is a subtype of sarcoma that occurs more commonly in the great vessels and pulmonary veins but rarely involves the heart (2).

\section{Case report}

A 37-year-old male with a past medical history of hypothyroidy presented to the emergency department with complaints of progressive exertional dyspnea (NYHA class II-III), anorexia and epigastric pain for a couple of days. The laboratory test results were unremarkable. Transthoracic echocardiogram (TTE) and transesophageal echocardiogram (TEE) revealed the presence of a left atrial mass of $40 \mathrm{X} 26 \mathrm{~mm}$ originating from the interatrial septum and causing a dynamic obstruction of the mitral valve. The mass was attached by a narrow pedicle of $4 \mathrm{~mm}$ localized on the interatrial septum between the fossa ovalis and the insertion of the anterior mitral leaflet (Fig. 1). Cardiac magnetic resonance imaging (MRI) confirmed the presence of a homogeneous atrial mass with isosignal intensity on T1-weighted images and increased T2 signal, without contrast enhancement after Gadolinium injection (Fig. 2). The most likely diagnosis for this mass was a large myxoma and the patient was scheduled for a surgical resection without delay.

Surgery was performed under a minimally invasive thoracoscopic approach (small incision in the right $4^{\text {th }}$ intercostal space, 2 percutaneous venous cannulas respectively inserted in the right internal jugular vein and in the right femoral vein). A transseptal approach via the right atrium was preferred in order to resect part of the interatrial septum if needed. After incision of the interatrial septum, we discovered a reddish, slightly gelatinous mass, attached to the lower part of the interatrial septum by a small pedicle (Fig. 3). The pedicle 
was resected with a small patch of the interatrial septum and the mass was removed. The interatrial septum was closed directly without interposition of a patch. Postoperative course was uneventful. Gross pathology of the operative specimen showed an encapsulated mass of 4.3 X 3.2 X $2.4 \mathrm{~cm}$ with soft consistency. Microscopic examination revealed the presence of a myxoid matrix with some cellular zone, in which spindle or stellar cells are identified (Fig. 4). Some nuclear atypias were present, however without abnormal mitotic figures. On this basis, pathologic analysis confirmed the diagnosis of cardiac myxoma. Patient was discharged from the hospital by day 7 . One month after hospital discharge, the patient was rehospitalized for bronchopneumonia and atrial fibrillation (AF). The cardiac imaging by TTE and thoracic CT performed at this time did not show any evidence of cardiac mass recurrence. Patient was treated by antibiotics and antiarrhythmic drugs with favorable clinical course. Four months after surgery, patient experienced recurrent AF and the routine TTE showed a recurrence of a left atrial mass of 26 X21 mm, with irregular shapes. TEE and cardiac MRI confirmed the features of the mass that is attached to the atrial septum at the same place as the previous tumor (Fig. 5). Although the recurrence occurred early, the presumptive diagnosis at this moment was a recurrent left atrial myxoma. A surgical resection of this recurrent left atrial mass was performed under median sternotomy and cardiopulmonary bypass. Due to recent history of paroxysmal AF, a concomitant surgical AF ablation procedure (Cox-Maze 4) was performed in the same operative time. A yellowish, slightly lobulated mass was removed, excising part of the interatrial septum (Fig. 6). The defect in the atrial septum was closed using a pericardial patch. Macroscopic examination of the specimen demonstrated a mass of soft consistency measuring $3.2 \times 3 \times 2.6 \mathrm{~cm}$. Histology showed an edematous stromal tissue with area of moderate to high cellularity. In these areas, packed spindle cells and stellar cells are found with fascicular growth pattern. The tumor cells had pleiomorphic, polylobed nuclei with frequent abnormal mitotic figures. Immunohistochemical analysis revealed an intense expression of the MDM2 oncogene in tumor cell nuclei, confirming the diagnosis of intimal sarcoma (Fig. 7). Adjacent atrial septum and resection margins were free of tumor. Early postoperative evolution was favorable and patient was discharged home by day 9. An adjuvant chemotherapy with doxyrubicin was administered for a total of 6 cycles. A metastasis in the left adrenal gland was discovered on the PET-CT at 1-year follow-up. Patient underwent a successful surgical resection of this metastasis and pathology confirm that it was a metastasis of the intimal sarcoma. An additional adjuvant chemotherapy with ifosfamide was given for 6 cycles and the patient is still in complete remission 18 months after the first operation.

\section{Discussion}

Cardiac sarcoma are rare malignant tumors that usually occurs in the $3^{\text {rd }}$ and $4^{\text {th }}$ decade of life with a male predisposition (sex ratio 2.5:1) (3). Primary intimal sarcoma of the heart is subtype of sarcoma that arises from subendothelial cells in the intima of great blood vessels. This tumor of mesenchymal origin rarely involves the heart and, when it occurs, the left atrium and the mitral valve are more often affected (1).

As other cardiac malignancies, they can cause valvular or inflow-outflow tract obstruction, thromboembolism, arrhythmias, or pericardial disorders or be totally asymptomatic and discovered incidentally (4).

In this report, the initial pathologic diagnosis of myxoma is questionable. The histopathological analysis showed the presence of a myxoid stroma, spindle and stellar cells, the absence of necrosis and of identifiable mitotic figures which is consistent with the diagnosis of myxoma. In fact, the main concern was about the presence of nuclear atypias, which is not a usual pattern in case of myxoma and which could point towards a malignant nature of the initial tumor. Indeed, since cardiac intimal sarcoma are infrequent, initial pathological and radiological features might be confused with those of other malignant or even benign cardiac tumors (5).

Another hypothesis to explain the initial diagnosis of cardiac myxoma is that the intimal sarcoma has arisen from residual myxoma cells in the interatrial septum. Some authors have reported the possibility of malignant transformation of cardiac myxoma $(6,7)$. This notion is however questioned by others who suggest that the malignant nature of the recurrent tumor is not always well defined (8). In our report, the malignancy and the histologic subtype of the second tumor is well established as the pathologic diagnosis was confirmed by immunohistochemical analysis (MDM2 overexpression) and by the development of a histologically proven 
metastatic disease.

The complete surgical resection with achievement of tumor free margin is the mainstay of the treatment but almost $50 \%$ of the patients are not suitable for this kind of resection due to the localization of the tumor (2). Intimal cardiac sarcoma is a highly aggressive tumor with a mean survival ranging from 3 months to 1 year. The life expectancy is twice as long for patients with complete surgical resection compared to patients with no resection (9). Other treatment modalities include radiation therapy and chemotherapy. Adjuvant therapy is usually offered to patients with surgically resected cardiac intimal sarcoma as recommended for soft tissue sarcomas (10). Cardiac transplantation is rarely considered as a treatment option because of the aggressive character of the intimal sarcoma and the high probability of distant micrometastasis at the time of diagnosis (11). In our case, despite a satisfactory local control after the second surgery, patient quickly developed a systemic metastasis, confirming that the cardiac transplantation was not indicated.

\section{Ethics statement}

Approval for publication was obtained from the patient and from our Institutional Review Board.

Legend Figure 1

Transesophageal echocardiogram. (A) Bicaval view demonstrating the left atrial mass attached to the atrial septum. (B) Mid esophageal $120^{\circ}$ view showing the dynamic obstruction of the mitral valve by the mass. RA, right atrium; LA, left atrium; LV, left ventricle.

\section{Legend Figure 2}

Four chamber post-contrast cardiac magnetic resonance imaging revealing the left atrial mass (asterisk). LA, left atrium; LV, left ventricle.

Legend figure 3

Thoracoscopic view of the reddish and slightly gelatinous mass attached to the left side of the interatrial septum. LA, left atrium; RA, right atrium; IA septum, interatrial septum; Asterisk, mass.

Legend figure 4

Scattered stellate and spindle cells without abnormal mitotic figures in abundant loose myxoid matrix suggesting the diagnosis of cardiac myxoma (Stain: $\mathrm{H} \& \mathrm{E}, \times 10)$.

Legend figure 5

Post- contrast cardiac MRI showing the recurrence of the left atrial mass attached to the interatrial septum in 4 chambers view (A) and short axis view (B). Asterisk, mass.

Legend figure 6

Resected yellowish, slightly lobulated left atrial mass with a part of the atrial septum

Legend figure 7

(A) Histopathology pictures of cardiac intimal sarcoma showing spindle cells in fascicles with nuclear pleomorphism and mitotic figures (Stain: H\&E, $\times 10$ ). (B) Immunohistochemistry profile shows positivity for MDM2.

\section{References}

1. Thuraisingam A, Fletcher N, Mckay G. Primary Cardiac Intimal Sarcoma. Heart, Lung and Circulation. 2019;28:S104.

2. Valecha G, Pau D, Nalluri N, Liu Y, Mohammad F, Atallah JP. Primary Intimal Sarcoma of the Left Atrium: An Incidental Finding on Routine Echocardiography. Rare Tumors [Internet]. 2016 Nov 17 [cited 2020 Jul 5];8(4). Available from: https://www.ncbi.nlm.nih.gov/pmc/articles/PMC5136772/ 
3. Ogechukwu C-N, Christopher N, Christoph S, Etinosasere O, Jose B. The Insidious Cardiac Tumor: A Primary Left Atrium Intimal Cardiac Sarcoma in a Young Patient [Internet]. Vol. 2019, Case Reports in Cardiology. Hindawi; 2019 [cited 2020 Jul 9]. p. e7245676. Available from: https://www.hindawi.com/journals/cric/2019/7245676/

4. Hudzik B, Miszalski-Jamka K, Glowacki J, Lekston A, Gierlotka M, Zembala M, et al. Malignant tumors of the heart. Cancer Epidemiol. 2015 Oct;39(5):665-72.

5. Marques Mendes E, Ferreira A, Felgueiras P, Silva A, Ribeiro C, Guerra D, et al. Primary intimal sarcoma of the left atrium presenting with constitutional symptoms. Oxf Med Case Reports [Internet]. 2017 Jul 5 [cited $2020 \mathrm{Jul} 8$ ];2017(7). Available from: https://www.ncbi.nlm.nih.gov/pmc/articles/PMC5497514/

6. Nguyen A, Awad WI. Cardiac Sarcoma Arising From Malignant Transformation of a Preexisting Atrial Myxoma. Ann Thorac Surg. 2016 Apr;101(4):1571-3.

7. Wang Bin, Li He, Xie Yuji, Cai Jie, Zhang Li, Li Yuman, et al. Undifferentiated Cardiac Sarcomas Originating From Malignant Transformation of Atrial Myxoma. Circulation: Cardiovascular Imaging. 2020 May 1;13(5):e010277.

8. Leduc C, Maleszewski JJ. Misconceptions About Malignant Metamorphosis in Myxoma. The Annals of Thoracic Surgery. 2016 Nov 1;102(5):1766.

9. Burke AP, Cowan D, Virmani R. Primary sarcomas of the heart. Cancer. 1992 Jan 15;69(2):387-95.

10. Adjuvant chemotherapy for localised resectable soft-tissue sarcoma of adults: meta-analysis of individual data. The Lancet. 1997 Dec;350(9092):1647-54.

11. Li H, Yang S, Chen H, Yang Z, Hong T, Hou Y, et al. Survival after heart transplantation for nonmetastatic primary cardiac sarcoma. Journal of Cardiothoracic Surgery. 2016 Oct 3;11(1):145.
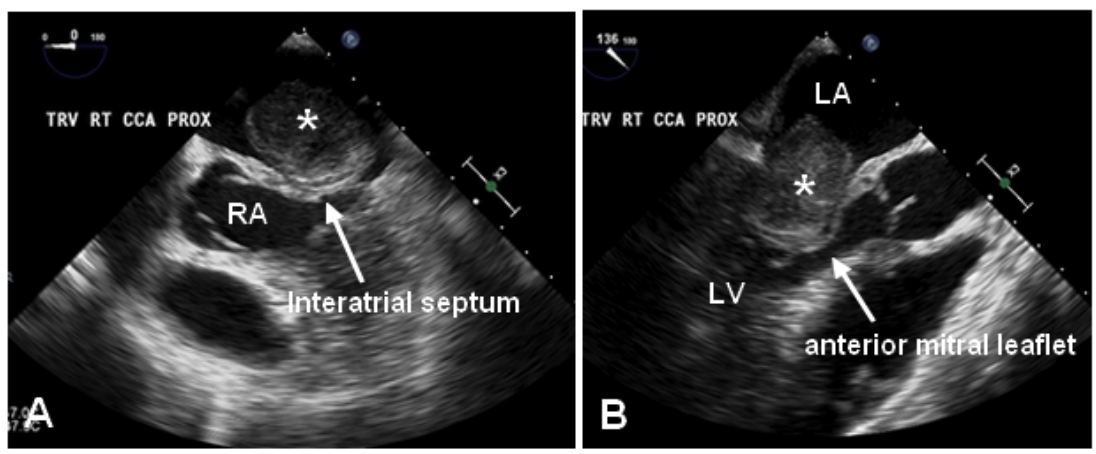

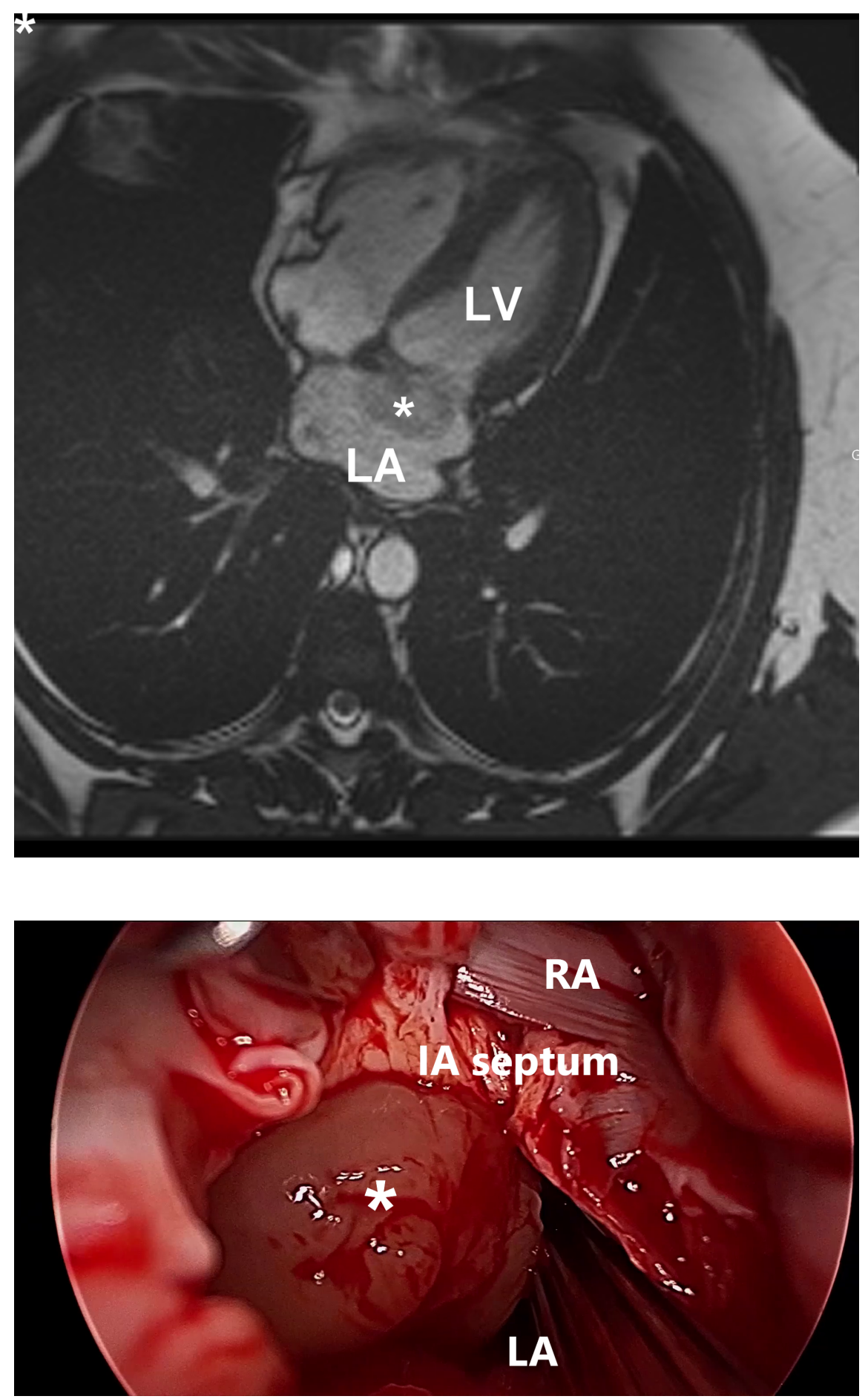

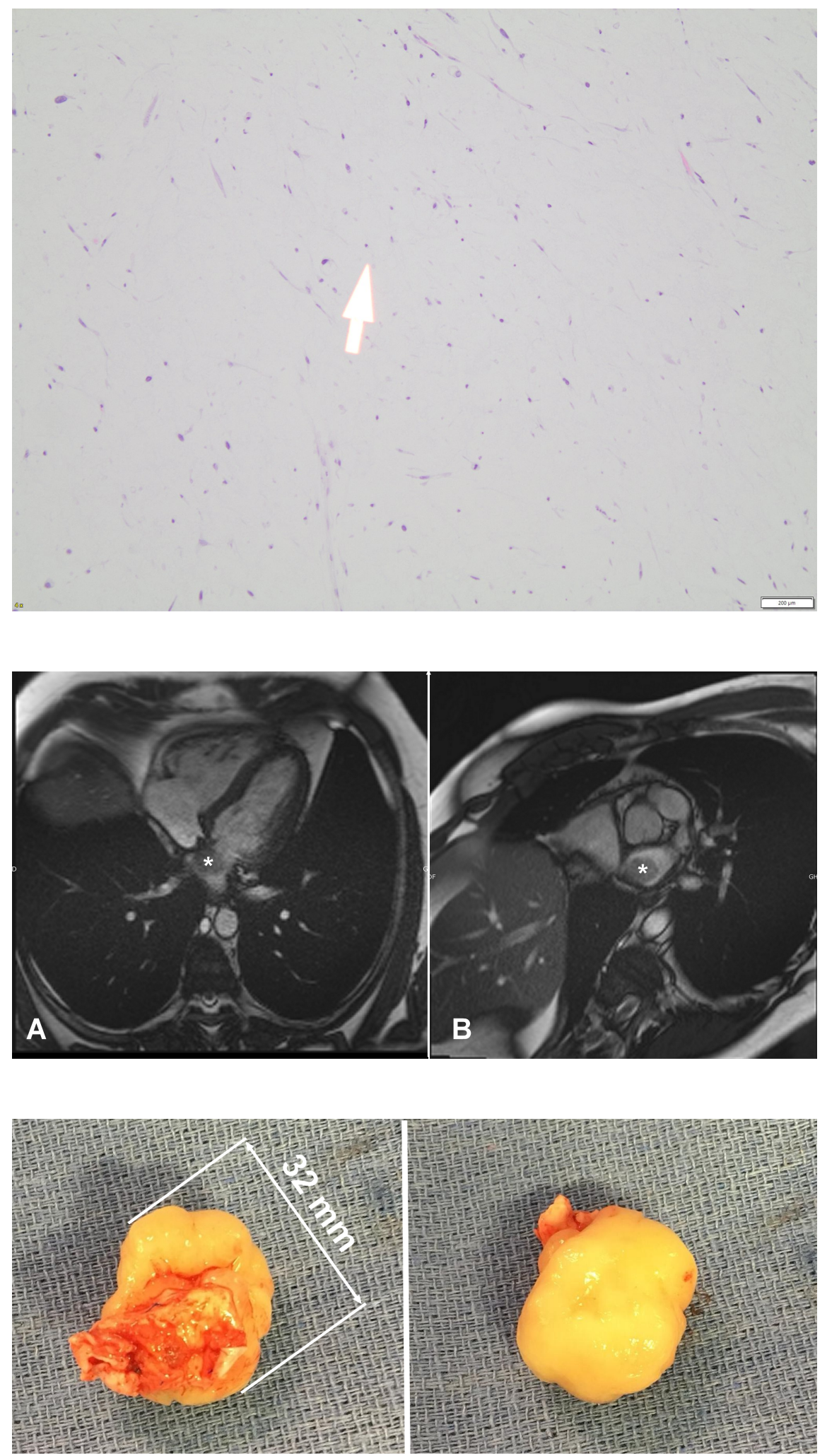


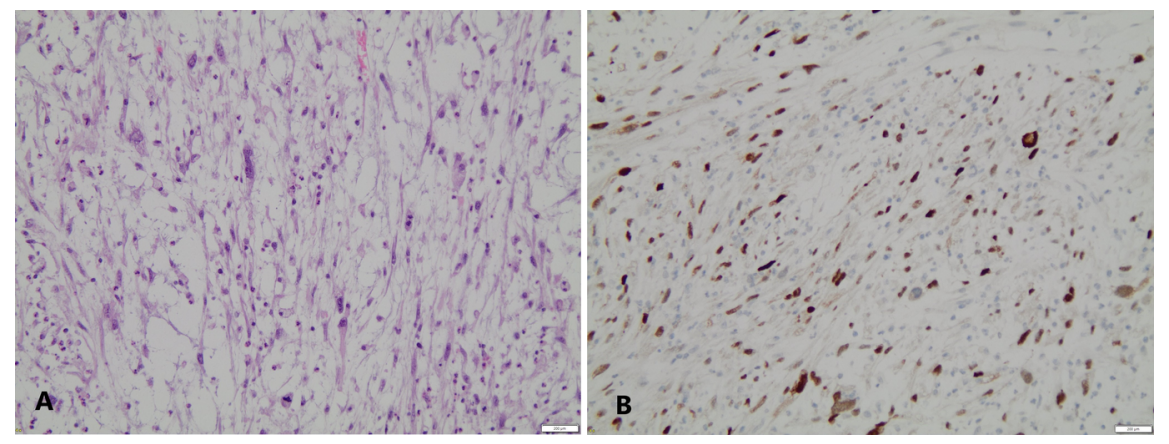

